\title{
Successful Surgical Management of Aortopulmonary window with Severe Pulmonary Artery Dilatation causing compression of Left Main Coronary Artery in an Adolescent
}

\author{
Anand Mishra ${ }^{1}$, Sanjeev Naganur ${ }^{1}$, Parag Barwad ${ }^{1}$, RUCHIT PATEL ${ }^{1}$, Vinay Upadhyay $^{2}$, \\ Vidur Bansal ${ }^{2}$, and Pratyaksha Rana ${ }^{1}$ \\ ${ }^{1}$ Post Graduate Institute of Medical Education and Research \\ ${ }^{2}$ PGIMER
}

October 12,2020

\begin{abstract}
Aortopulmonary Window is a rare congenital heart defect comprising $0.1-0.2 \%$ of the total spectrum. Spontaneous closure of this defect is unknown and survival into childhood and adult life is rarely seen. Severe pulmonary artery hypertension develops and causes dilatation of the pulmonary artery. Left main coronary artery extrinsic compression by an enlarged pulmonary artery is a rare complication and a potential cause for chest pain and sudden cardiac death in patients with pulmonary hypertension.
\end{abstract}

\section{INTRODUCTION}

Aortopulmonary window(APW) is a rare congenital cardiac anomaly, occurring due to incomplete separation of conotruncus by the aorticopulmonary septum with separate semilunar valves. The usual presentation is in early infancy and childhood due to congestive heart failure. ${ }^{1}$ Early surgical closure is required before progression to irreversible pulmonary hypertension beyond infancy, which contradicts closure. Recently, attempts have been made for transcatheter device closure of this anomaly, but it is not possible in all cases, particularly for large proximal and distal types. Surgical closure is feasible for all types with excellent longterm outcomes. ${ }^{2}$ Here we present the case of a 14-year-old boy, with a large type-I APW who developed severe pulmonary artery hypertension causing the dilated pulmonary artery to cause compression of the left main coronary artery. He underwent a successful anterior sandwich repair after a failed attempt at device closure.

\section{CASE SUMMARY}

A 14 years of age boy presented to the cardiologist with complaints of dyspnea and angina on exertion and palpitations for the past one year. Clinically, there was no cyanosis. Chest X-Ray showed cardiomegaly with enlargement of left-side chambers of the heart and a prominent main pulmonary artery(MPA) impression. His resting ECG was essentially normal with no ST-T changes. On echocardiography(Figure-1), there was an enlarged Left atrium, Left ventricle and MPA with severe pulmonary artery hypertension(PAH). There was a large type-I APW of $24 \mathrm{~mm}$. CT-Angiography(Figure-2b) was done which confirmed the above findings. On the catheterization study(Figure-2a), pulmonary artery pressures were at systemic levels but reversible with $100 \%$ Oxygen. MPA was dilated $(3.5 \mathrm{~cm})$ and was seen compressing the left main coronary artery(LMCA). The closure was attempted with a $24 \mathrm{~mm}$ VSD device and $34 \mathrm{~mm}$ ASD device(Amplatzer St.Jude Medical, USA) but was unsuccessful despite multiple attempts. Also, there were transient ST-T changes while deploying the device, hence the procedure was abandoned and was referred for surgical closure of APW. Under normothermic cardiopulmonary bypass, aortic root cardioplegia was given after snaring 
both pulmonary arteries with a LV vent in-situ. A trans-window vertical incision was used for opening the defect(Figure-3a, Supplementary-Video-1). The right pulmonary artery(RPA) (Figure-3b) and coronary ostia(Figure-3c) were away from the defect. Polytetrafluoroethylene(PTFE) patch was used to close the window with the anterior sandwich technique. The LMCA was seen arising eccentrically to the left and a little higher than usual but the caliber was found to be adequate on putting a Garrett probe(?Scanlan Memory Garett). Post cardiopulmonary bypass, PA was found to be soft, less distensible and PA pressures were $50 \%$ of the systemic pressures. The postoperative course was uneventful and the patient was discharged on day 5 .

\section{DISCUSSION}

Early intervention and surgical closure is mandatory before it leads to irreversible PAH. APW may occur as an isolated lesion, or it can be associated with other cardiac abnormalities in one-third to one-half of cases. ${ }^{1}$ Echocardiography usually establishes diagnosis in most cases. Assessment of operability can be determined by the clinical status, absence of cyanosis, cardiomegaly on chest X-rays, features of left ventricular volume overload on electrocardiogram and cardiac catheterization findings. ${ }^{2}$

Still, presentation beyond infancy is not so uncommon in developing countries due to poor socioeconomic conditions. ${ }^{3}$ In the presence of severe $\mathrm{PAH}$, if significant reversibility in the pulmonary vascular resistance index could be demonstrated with oxygen, the condition can be successfully corrected with good longterm outcomes. Mori and colleagues ${ }^{4}$ divided APW into three types; types I-III. The classification scheme used by the Society of Thoracic Surgeons adds a fourth 'intermediate' category, suggesting a continuum of morphologies. ${ }^{5}$ Severe PA dilatation has been described as a predisposing risk factor for LMCA compression in patients with PAH. ${ }^{6}$ The pulmonary artery diameter is the most important aspect of the LMCA stenosis in these cases, but other aspects like mean pulmonary artery pressure or angina symptoms are not significantly associated with coronary compression. ${ }^{6}$

It is important to confirm myocardial ischemia due to LMCA compression before indicating a treatment. ${ }^{7}$ There are patients in whom the hemodynamics can be stabilized only by treating the cause of pulmonary hypertension. Although PCI and rarely CABG may be an option, but as in our case, just treating the cause of the massive PA dilatation could help resolve the compression of LMCA.

In the presence of reversible pulmonary vascular disease, surgical correction is indicated and may result in a regression of both symptoms and PAH. The evolution of the surgical management of APW has progressed from off-pump ligation toward patch repair. Simple ligation of APW has a risk of a residual defect and is justified for small defects only.

The intermediate type, which has adequate superior and inferior rims, is best suited for device closure. Device closure of APW in adolescents is rarely reported because of the rarity of the defects with good margins, associated congenital cardiac anomalies requiring cardiac surgery, early development of Eisenmenger syndrome in this population or due to technical challenges precludes device closure. ${ }^{8}$ Our case was a type-I APW with no other associated cardiac anomaly. We approached through the window for surgical closure. It provides excellent exposure of the defect along with a clear view of ostia of LCA, RCA and RPA. It isn't associated with semilunar valves distortion and post-surgical great arteries stenosis. With a single PTFE patch, the posterior part of the defect was closed with suturing at the posterior aortic wall. For the anterior part, the anterior sandwich approach ${ }^{9}$ was used taking bites from the aorta, patch and through the pulmonary artery anterior wall at the defect level.

\section{CONCLUSION}

Though a rare congenital cardiac anomaly, presentation of the aortopulmonary window in the adolescent age group is observed among developing countries. Irreversible pulmonary artery hypertension precludes closure of the defect. Device closure is restricted for intermediate types with adequate superior and inferior rims. Surgical closure can be safely achieved for reversible pulmonary hypertension cases with excellent long-term outcomes. However, given the low prevalence of extrinsic compression of the LMCA due to PAH, a large 
multicentre trial is needed to identify the optimal treatment.

\section{REFERENCES}

1. Backer CL, Mavroudis C: Surgical management of aortopulmonary window: A 40-year experience. Eur J Cardiothorac Surg 2002;21:773-779.

2. Aggarwal SK, Mishra J, Sai V, Iyer VR, Panicker BKP. Aortopulmonary window in adults: Diagnosis and treatment of late- presenting patients. Congenit Heart Dis 2008;3:341-46.

3. Kothari SS. Pediatric cardiac care for the economically disadvantaged in India: problems and prospects. Ann Pediatr Cardiol 2009;2:95-8.

4. Mori K, Ando M, Takao A, et al. Distal type of aortopulmonary window: Report of four cases. Br Heart J 1978;40:681-689

5. Jacobs JP, Quintessenza JA, Gaynor JW, et al. Congenital Heart Surgery Nomenclature and Database Project: aortopulmonary window. Ann Thorac Surg 2000;69(suppl):S44-49

6. Mesquita SM, Castro CR, Ikari NM, Oliveira SA, Lopes AA. Likelihood of left main coronary artery compression based on pulmonary trunk diameter in patients with pulmonary hypertension. Am J Med 2004;116:369-374.

7. Pina Y, Exaire JE, Sandoval J. Left main coronary artery extrinsic compression syndrome: a combined intravascular ultrasound and pressure wire. J Invasive Cardiol. 2006;18:E102-4.

8. Trehan V, Nigam A, Tyagi S. Percutaneous closure of nonrestrictive aortopulmonary window in three infants. Catheter Cardiovasc Interv. 2008;71:405-11.

9. Johansson L, Michaelsson M, Westerholm CJ, Aberg T. Aortopulmonary window: a new operative approach. Ann Thorac Surg 1978;25:564-7

\section{FIGURE CAPTIONS}

Fig-1 -2D-Echocardiography-(a)Parasternal short axis view showing large APW (red arrow) with bidirectional flow,(b) M-mode showing dilated LV(LVID in diastole Z-score of +3.5 and systole of +3.4 ), (c)Parasternal short axis view showing suspicion of LMCA compression in(yellow arrow), LAD(red arrow appears bigger), (d)Suprasternal view showing DTA flow reversal in $1 / 3^{\text {rd }}$ of diastole consistent with aortic run off, suggesting operable hemodynamics

Fig-2 -(a)Cardiac Catheterization showing the APW with the compression of the LMCA by dilated MPA (marked by an arterisk), (b)CT-Angio showing the same.

Fig-3 -Intra-Operative pictures (a)Intra-operative anatomy of the heart showing the APW and the dilated MPA, (b)On opening the APW with a probe in RPA, (c) On opening the APW with a probe in LMCA 

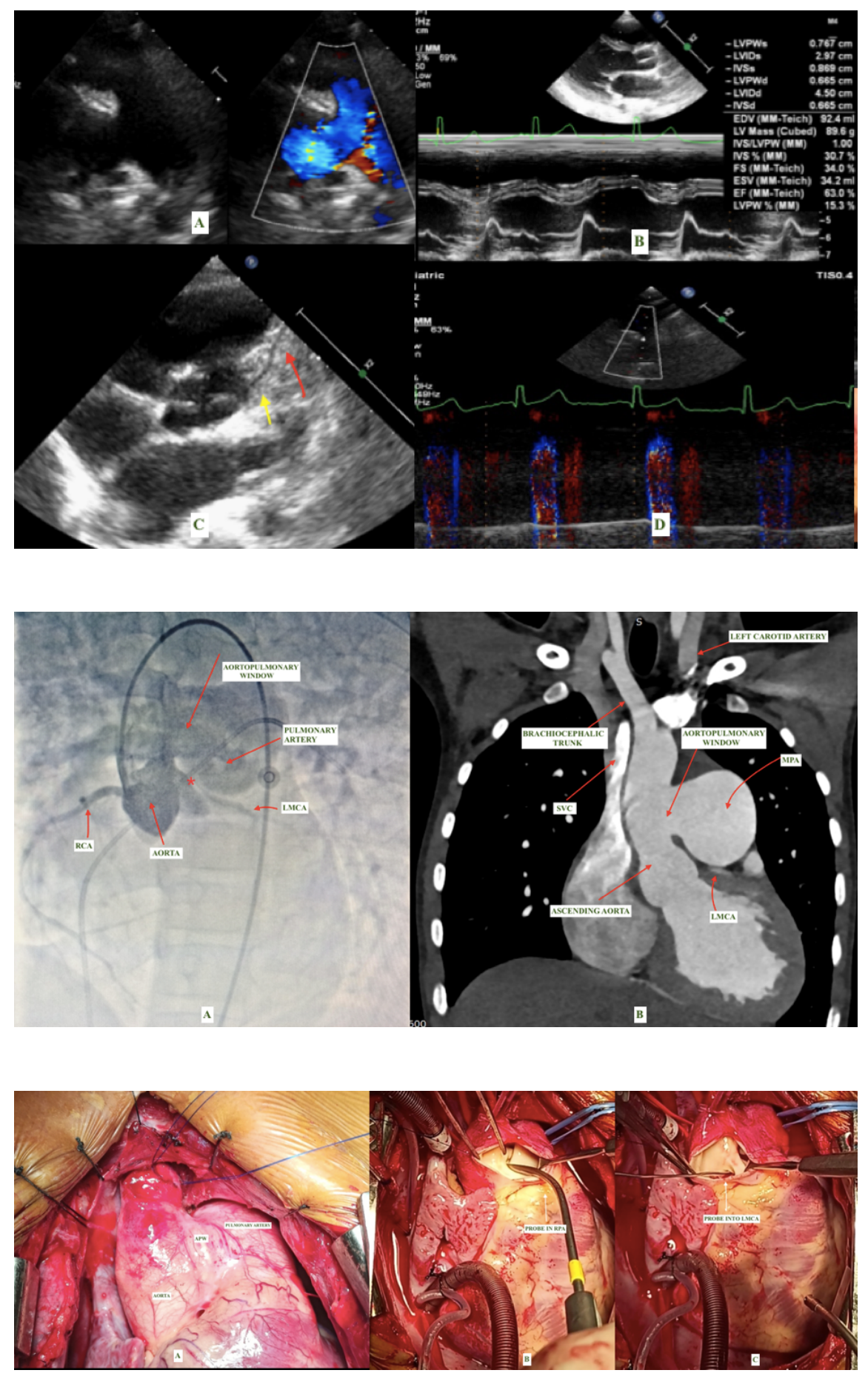